\title{
Treating the violent and psychotic patient: A clinical guide to potential cardiac complications
}

\author{
Nicole Renee Graham¹, Garth Nigel Graham² \\ 1. Department of Psychiatry, School of Medicine, Yale University, New Haven, USA. 2. Department of Medicine, School of \\ Medicine, University of Florida, Gainesville, FL, USA.
}

Correspondence: Garth Nigel Graham. Address: Department of Medicine, School of Medicine, University of Florida, Gainesville, FL, USA. Email: Garth.Graham@medicine.ufl.edu

Received: November 26, 2013

Accepted: January 6, $2014 \quad$ Online Published: February 7, 2014

DOI : $10.5430 /$ jnep.v4n4p114

URL: http://dx.doi.org/10.5430/jnep.v4n4p114

\begin{abstract}
In effectively treating the violent and psychotic patient, medical professionals are charged with the task of mitigating harm to all involved. While non-pharmacologic methods are often employed first, chemical restraint may also be necessary. The agents most often utilized in the management of such patients include antipsychotics - first and second generation agents - and benzodiazepines. When considering use of these agents, it is prudent that nurses appreciate the potential risks, including those of a cardiac nature given that they represent some of the most dangerous. This review article aims to highlight the potential cardiac complications and provide guidance in navigating this important task of harm mitigation for the violent and psychotic patient.
\end{abstract}

\section{Key words}

Psychosis, Cardiac care, Antipsychotics, Benzodiazepines

\section{I ntroduction}

Effectively treating the violent and psychotic patient can present a challenge for nurses and other medical providers. Hostile and self-destructive behaviour is a very common reason for patients to present to the emergency room and for subsequent admission to the hospital ${ }^{[1]}$. On-going violent behaviour can prolong the duration of a hospital stay and serve as a serious impediment for subsequent discharge or community placement ${ }^{[2]}$. Often, such risk of violence necessitates the efforts of the entire treatment team, who are charged with the task of mitigating harm.

The vast majority of psychiatric patients are not violent, and in fact only a small minority account for an excessively high number of events ${ }^{[3]}$. Nurses and other clinicians are more likely to encounter the violent patient in certain types of clinical settings including locked wards, forensic units, and psychiatric intensive care units. Prior to admission to one such unit, these patients often present to the emergency department and the staff there have been shown to be at increased risk for assault and other forms of harm ${ }^{[4]}$.

In addition to the clinical impact of dealing with the individual violent patient this issue has an overall public health impact that requires strategic thinking by nurses and other clinicians. Although the treatment of the violent patient is a relatively frequent issue encountered in clinical practice there have been relatively few studies looking at effective treatment 
strategies and guidelines for managing aggressive and violent patients and the side effects of the medications that may be employed ${ }^{[5]}$.

In the acute emergency setting, violence risk is often managed via the use of psychotropic medications, physical restraints, seclusion, and/or the removal of noxious stimuli. While these other forms of de-escalation are important and should be tried first in most cases ${ }^{[6]}$, this article will focus on the use of psychotropic medications in managing the violent and psychotic patient and in particular understanding the cardiac complications of these medications.

Given the nature of the patient and location, nurses are the front line agents in helping to develop an appropriate treatment strategy; in many cases this strategy will require quick thinking and appreciation of the potential side effects of those agents most often used in managing such patients. Of the potential side effects, cardiac are considered amongst the most important to be aware of given the risk to the patient and the quick response necessary should they occur.

When chemical restraint is necessary in managing the violent patient, the medications typically utilized include antipsychotics and benzodiazepines either in combination or via mono-therapy. An example of such a "cocktail" used to mitigate the violence risk in the emergency setting includes antipsychotics such as haloperidol or risperidone and a benzodiazepine such as lorazepam or diazepam. One such treatment algorithm is shown in Figure $1^{[7]}$, as is evident, administration of intramuscular (IM) medications is usually reserved for instances in which oral administration of the medication(s) is refused or otherwise impossible.

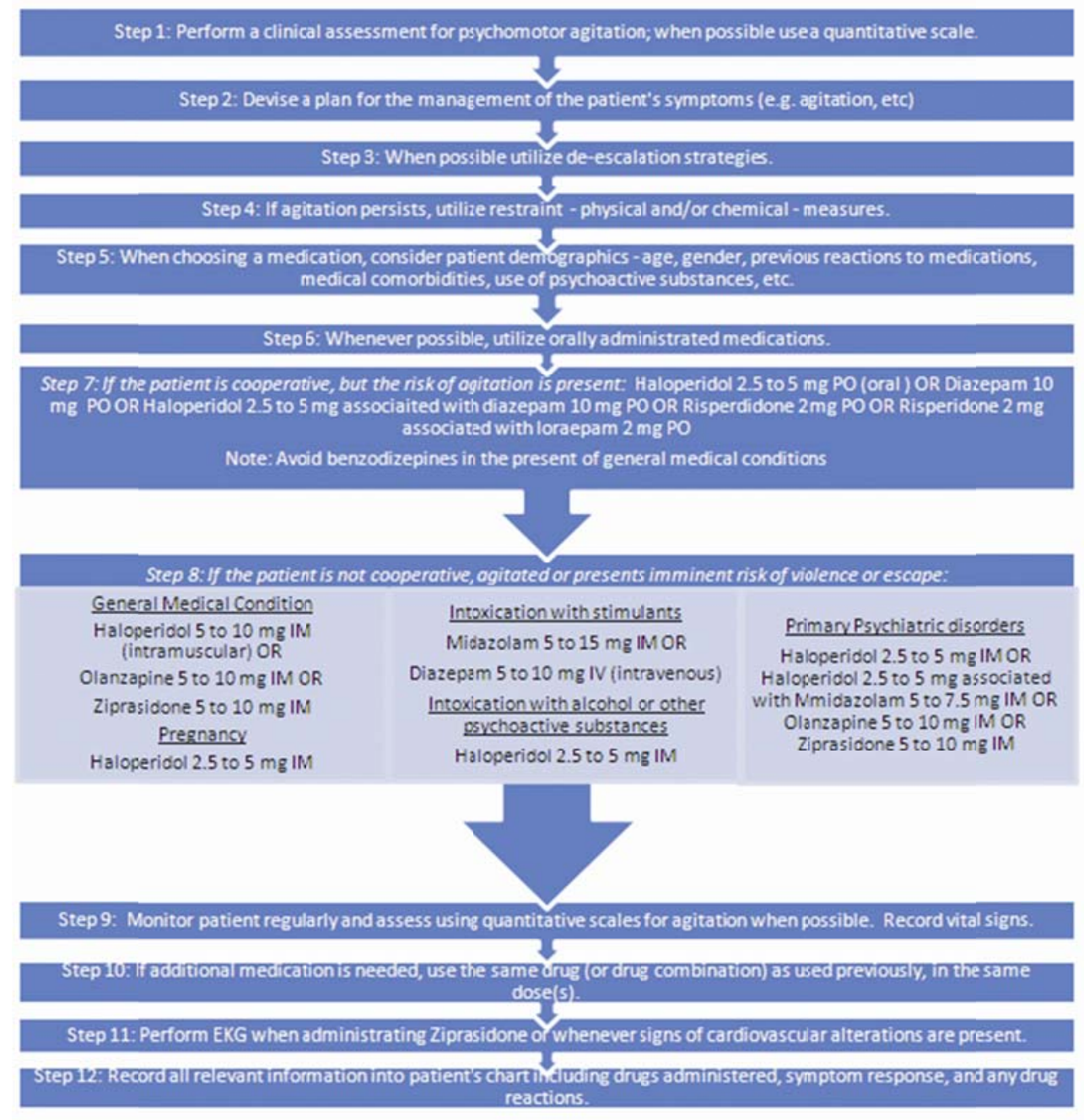

Figure 1. A pharmacologic treatment algorithm for treating the violent and psychotic patient. Formatting Published by Sciedu Press 
When selecting which medication(s) to use, many factors come into play. As is outlined in Figure 1, patient demographics, presence of agitation, prior reactions to medications, specific side effect profiles, and desired route of drug administration should be considered. Amongst the antipsychotics, there are two classes from which a medication can be selected. Typical, or first generation, antipsychotics include older medications such as Haloperidol, and characteristically have more extrapyramidal side effects than the newer, atypical or second generation medications. Table 1 includes more examples of first and second generation agents. In addition to the aforementioned classification, antipsychotics can further be classified by their relative potency; Table 2 shows examples of high, mid and low for several first generation antipsychotics ${ }^{[8]}$. No more effective than low potency agents, high potency agents (e.g. fluphenazine) simply require a lower dose to exact their effects.

Table 1. Examples of typical (first generation) and atypical (second generation) antipsychotic medications.

\begin{tabular}{ll}
\hline Classification & Examples \\
\hline Typical/First Generation & Haloperidol, Fluphenazine, Perphenazine, Chlorpromazine, Thioridazine, Loxapine \\
Atypical/Second Generation & Clozapine, Risperidone, Ziprazidone, Olanzapine, Quetiapine \\
\hline
\end{tabular}

Table 2. Potency classification for first generation antipsychotic medications (high, mid, low) with examples listed.

\begin{tabular}{ll}
\hline Potency & Examples \\
\hline High & Haloperidol, Fluphenazine \\
Mid & Perphenazine, Loxapine \\
Low & Chlorpromazine, Thioridazine \\
\hline
\end{tabular}

\section{Heart disease and psychotropic medications}

Cardiac side effects of psychotropic medications are varied and in some cases can be life threatening. The risk to the violent and psychotic patient is even greater than that of the general population, as patients with serious mental illness are considered as a 'high risk' population with regard to cardiovascular morbidity and mortality ${ }^{[9,10]}$. The etiology of the increased risk of cardiovascular mortality and morbidity in these individuals is not entirely understood; they most likely represent an accrual of 'traditional' risk factors including obesity, metabolic syndrome, hyperlipidemia, smoking and others ${ }^{[11]}$. These risk factors are compounded in the potentially violent patient with a serious psychiatric illness ${ }^{[12]}$.

While the majority of the cardiac side effects for first generation antipsychotic agents are fairly well known, there is less familiarity with those associated with the newer second generation medications. Additionally, providers may not appreciate the potential complications of using a combination cocktail as is typically employed. For these reasons, it is imperative that nurses familiarize themselves with these very important cardiac risks.

Appreciation for how and where these medications work is a necessary part of reducing potential risk to patients. These medications act at a variety of receptors, some of which provide their intended therapeutic effects, including dopamine and serotonin, GABAA receptors (benzodiazepine) and histamine receptors; they also interact with muscarinic and alphaadrenergic receptors ${ }^{[13]}$. The interaction with these receptors both centrally and peripherally accounts for the nature of the cardiac side effects.

\section{Cardiac complications in treating the aggressive patient}

There are a number of notable complications that nurses and the clinical team should be aware of when treating the violent/aggressive patient with psychotropic medications, the most common of which include ${ }^{[14]}$ :

1) Hypotension 

2) Syncope
3) Bradycardia

The incidence of hypotension varies across classes of psychotropic drugs. Within the antipsychotics, the lower potency first generation drugs (e.g. Thioridazine or Chlorpromazine) have a higher association with hypotension than the higher potency drugs (e.g. Haloperidol). Additionally, first generation antipsychotics have a higher rate than the newer second generation drugs, with as high as $19 \%$ in the former and around $9 \%$ in the latter ${ }^{[15]}$. The occurrence of syncope also fluctuates across these medication groups; it ranges from as high as $6 \%$ in patients treated with first generation antipsychotics to a mere $0.2 \%$ in Olanzapine treated patients and $1.1 \%$ in Ziprazidone treated patients ${ }^{[16]}$. While these risks may seem rather low, the incidence of such side effects increases with more rapid forms of administration (e.g. IM) as is commonly seen when treating the violent psychotic patient.

In addition to syncope and hypotension, more life threatening cardiac complications of these medications can also occur. These include the following ${ }^{[17]}$ :

1) QT prolongation,

2) Ventricular arrhythmias

3) Sudden Cardiac Death

Patients with a prior history of a prolonged QT interval on their ECG are at greater risk for developing further prolongation of their QT interval. The higher the degree of QT prolongation the higher the risk but ventricular arrhythmias are less likely to occur with a QT less than 500ms and one major study showed a mean QT of 580ms prior to the onset of serious ventricular arrhythmias ${ }^{[18]}$. These can degenerate into polymorphic ventricular arrhythmia otherwise known as torsade's de pointes and a small proportion of these may go on to ventricular fibrillation and even to sudden cardiac death ${ }^{[19]}$. Given the serious nature of these complications it is important for nurses to be able to identify the symptoms in an already violent and agitated patient. These symptoms include a complaint of palpitations, new onset seizure activity, loss of consciousness or any other change on mental status. Nurses and other members of the team need to be vigilant of these symptoms and what they may represent.

\section{Measuring the QT interval}

Given the extreme risk associated with QT prolongation it is important to understand how to properly assess this parameter. The QT interval is measured in milliseconds from the start of the Q wave to the end of the T wave on the ECG. It reflects the time for depolarization and then repolarization of the ventricle of the heart. When the heart rate increases the QT interval gets shorter and when the heart rate decreases the QT interval gets longer. It is often impossible to get a good ECG in an agitated patient; it is advised in this setting to try and get a copy of prior ECGs if possible on these patients and be aware of the risks associated with a QT more than 500ms. Other risk factors for a prolonged QT interval include hypocalcemia, hypomagnasemia, hypokalemia, prior bradycardia, left ventricular hypertrophy and psychiatric illnesses such as anorexia nervosa.

\section{Risk factors for cardiac complications}

The elderly, those with autonomic dysfunction and patients with underlying cardiovascular disease are at increased risk for complications such as hypotension, bradycardia and syncope. This is especially important to understand when treating the elderly demented patient who might be experiencing a psychotic episode. These patients might acutely develop dizziness, light-headedness or double vision related to hypotension or relative hypotension secondary to a medication cardiac effect. 
In addition to the above, particular care must be taken in the use of atypical (second generation) agents in the demented elderly, as there is an increased risk of stroke in these patients. Caution must be taken in these patients, particularly in patients with a prior history of stroke or transient ischemic attack (TIA), as they have up to a threefold increased risk of stroke. Because of these major risks, regulatory guidance has pointed to the use of these atypical medications as potentially a "rescue" form of medication (i.e. in emergency situations only) in the aggressive violent patient with dementia and a history of cerebrovascular disease ${ }^{[20]}$. Despite these warnings, a survey of expert opinion of 53 US based experts (38 geriatric psychiatrists and 14 geriatric internists) identified risperidone as the most common first line agent in agitated/ violent patients with dementia and delusions ${ }^{[21]}$.

Even though all antipsychotic medications have a potential for causing ECG abnormalities and subsequent ventricular arrhythmias the risk is noted to be higher with certain drugs including Thioridazine and Ziprazidone ${ }^{[22]}$. Although there are no randomized trials comparing the risks between drugs it is thought that drugs such as haloperidol are the safest in terms of overall risk. It is also worth noting that tricyclic antidepressants might increase the level of some of these drugs through its interaction with the liver enzyme cytochrome P450.

\section{Cardiac concerns of using Benzodiazepines in the acute setting}

In addition to the use of antipsychotics to treat aggression and violence in the emergency situation, benzodiazepines play an important role. Benzodiazepines, most notably lorazepam, are often used as either mono-therapy or in combination with an antipsychotic to help calm the violent and psychotic patient. As mentioned previously, these agents work at the GABAA receptors. They are generally well tolerated with the exception of a few important side effects which are related to excess amounts or a combination of sedatives or other central nervous system (CNS) depressants. The most common of these are non-cardiac and are related to over sedation and respiratory depression, but in this context, hypotension, bradycardia and possibly cardiac arrest can occur. While there are few studies on the cardiac risks of using a combination of the discussed medications, one study showed a $14 \%$ reduction in cardiac output with the use of diazepam alone ${ }^{[23]}$.

\section{Treatment of cardiac complications}

Cardiac complications including hypotension and other related effects occur more often after intramuscular administration or fast titration. Thus in the aggressive/violent patient where there is concern for negative cardiac side effects restraint in the initial dosing and speed of titration should be paramount. Factors such as hypovolemia, dehydration or low prior oral intake may also exacerbate symptoms as well as concomitant antihypertensive medications. In these cases rapid fluid administration may help alleviate symptoms once they develop.

There are a number of steps that can be taken to minimize the risk of QT related cardiac complications including electrolyte monitoring and replacement and being vigilant of possible drug-drug interactions as previously noted. Once ventricular arrhythmia has developed if the vital signs are stable then the ACLS stable tachycardia algorithm can be utilized including aggressive electrolyte repletion. If the arrhythmia is unstable with a polymorphic ventricular rhythm then unsynchronized defibrillation should be employed.

\section{Summary}

The aggressive or violent patient is often encountered in nursing practice. When these patients are encountered, effective treatment strategies may include the use of an antipsychotic and/or a benzodiazepine in reducing harm to those involved. The cardiac complications of these drugs may include hypotension, syncope, QT prolongation, ventricular arrhythmia and 
even rarely sudden cardiac death. Given the serious nature of these complications, nurses and other members of the treatment team must be aware of the potential complications, as well as strategies to prevent and treat their occurrence.

\section{References}

[1] Greenfield TK, McNiel DE, Binder RL. Violent behavior and length of psychiatric hospitalization. Hosp Community Psychiatry. 1989; 40: 809-814.

[2] Bigelow DA, Cutler DL, Moore LJ, McComb P, Leung P. Characteristics of state hospital patients who are hard to place. Hosp Community Psychiatry. 1998; 39: 181-185.

[3] Swanson J. Mental disorder, substance abuse, and community violence: an epidemiological approach. In Monahan JSteadman Heds. Violence and Mental Disorder: Developments in Risk Assessment. Chicago, Ill University of Chicago Press. 1994: 101-136.

[4] Petit JR. Management of the Acutely Violent Patient. Psychiatric Clin N Am. 2005; 28(3): 701-711.

[5] Volavka J, Citrome L. Atypical antipsychotics in the treatment of the persistently aggressive psychotic patient: methodological concerns. Schizophr Res. 1999; 35: S23- S33.

[6] Coburn VA, Mycyk MB. Physical and Chemical Restraints. Emerg Med Clin N Am. 2009; 27: 655-667.

[7] Mantovani C, Migon MN, Alheira FV, Del-Ben CM. Management of the violent or agitated patient. Rev Bras Psiquiatr. 2010; 32(Suppl 2): S96-S103.

[8] Schatzberg AF, Cole JO, DeBattista C. Manual of Clinical Psychopharmacology. 7th ed, Virginia: American Psychiatric Publishing, 2010, Chapter 4.

[9] Mackin P. Cardiac Side Effects of Psychiatric Effects. Hum Psychopharmac. 2008; 23(Suppl 1): 3-14.

[10] Barnett AH, Mackin P, Chaudhry I, Farooqi A, Gadsby R, Heald A, et al. Minimising metabolic and cardiovascular risk in schizophrenia: diabetes, obesity and dyslipidaemia. J Psychopharmacol. 2007; 21: 357-373.

[11] Atlantis E, Sullivan T. Changes in cardiovascular disease burden associated with psychopathology in Australian adults $2004-2008$. Gen Hosp Psychiatry. 2012; 34(4): 345-351.

[12] Curtis J, Newall HD, Samaras K. The heart of the matter: Cardiometabolic care in youth with psychosis. Early Interv Psychiatry. 2012; 6(3): 347-353.

[13] Ananth J, Parameswaran S, Gunatilake S. Side effects of atypical antipsychotic drugs. Curr Pharm Des. 2004; 10(18): $2219-29$.

[14] Brignole M, Alboni P, Benditt D, Bergfeldt L, Blanc, J-J, Block Thomsen, PE, et al. Guidelines on management (diagnosis and treatment) of syncope. Eur Heart J. 2001; 22: 1256-1306.

[15] FDA. 2001b. FDA Pharmacological Drugs Advisory Committee Briefing Document for Zyprexa Intramuscular (olanzapine for injection). 1-115.

[16] Newcomer JW. Second-generation (atypical) antipsychotics and metabolic effects: a comprehensive literature review. CNS Drugs. 2005; 19(Suppl. 1): 1-93.

[17] Koponen H, Alaräisänen A, Saari K, Pelkonen O, Huikuri H, Raatikainen MJ, et al. Schizophrenia and sudden cardiac death: a review. Nord J Psychiatry. 2008; 62(5): 342-345.

[18] Stratmann HG, Kennedy HL. Torsades de pointes associated with drugs and toxins: recognition and management. Am Heart J. 1987; 113: 1470-1482.

[19] Schouten EG, Dekker JM, Meppelink P, Kok FJ, Vandenbroucke JP, Pool J. QT interval prolongation predicts cardiovascular mortality in an apparently healthy population. Circulation. 1991; 84: 1516-1523.

[20] Bullock R. Treatment of behavioural and psychiatric symptoms in dementia: implications of recent safety warnings. Curr Med Res Opin. 2005; 21(1): 1-10.

[21] Alexopoulos GS, Streim J, Carpenter D, Docherty JP. Expert Consensus Panel for Using Antipsychotic Drugs in Older Patients. Using antipsychotic agents in older patients. J Clin Psychiatry. 2004; 65(Suppl 2): 5-99.

[22] Taylor D. Ziprasidone in the management of schizophrenia: the QT interval issue in context. CNS Drugs. 2003; 17(6): 423-430.

[23] Rao S, Sherbaniuk RW, Prasad K, Lee SJ, Sproule BJ. Cardiopulmonary effects of diazepam. Clin Pharmacol Ther. 1973; 14: 182-189. 